\title{
A metabolomics approach used to profile plasma from portal-arterial pigs revealed differences between breads incurred by dietary fibre and protein contents
}

\author{
Kirstine Lykke Nielsen ${ }^{1}$, Mette Skou Hedemann ${ }^{2}$, Helle Nygaard Lærke ${ }^{2}$, Henry Jørgensen ${ }^{2}$ and \\ Knud Erik Bach Knudsen ${ }^{2} *$ \\ ${ }^{1}$ Department of Forensic Medicine, Forensic Chemistry, Aarbus University, Brendstrupgårdsvej 100, DK-8200 Aarbus N, Denmark \\ ${ }^{2}$ Department of Animal Science, Aarbus University, Blichers Allé 20, DK-8830 Tjele, Denmark.
}

(Received 26 October 2013 - Final revision received 28 October 2013 - Accepted 2 April 2014)

Journal of Nutritional Science (2014), vol. 3, e18, page 1 of 10

doi:10.1017/jns.2014.14

Abstract

A liquid chromatography-MS (LC-MS) metabolomics analysis of plasma from portal-arterial catheterised pigs fed breads prepared with whole-grain rye or wheat flour with added concentrated arabinoxylan (AX) or $\beta$-glucan (BG) was conducted. Comparison of the effects of concentrated fibres with whole grains has received little attention. Six female catheterised pigs were given two white wheat breads with wheat AX or oat BG, two rye breads with ground rye (GR) or intact rye kernels (RK), and a control white wheat bread (WF) on separate occasions in a randomised cross-over design. The amount of available carbohydrate was similar for the five breads but varied in the content of protein. Plasma was collected continuously for $4 \mathrm{~h}$ after feeding. Glucose levels in the portal vein were reduced postprandially in response to the AX, GR and RK breads that had high contents of AX compared with WF bread $(P<0.03)$. AX and RK breads further tended to decrease plasma levels of some lysophosphatidylcholine species $(P \leq 0 \cdot 10)$. The abundance of amino acids in plasma correlated with the protein contents in the breads and leucine uptake significantly affected insulin secretion in the mesenteric artery. In conclusion, the present study revealed that concentrated AX in wheat bread had similar positive effects as whole-grain rye bread on glucose and lipid metabolism.

Key words: Arabinoxylan: $\beta$-Glucan: Catheterised pigs: Rye: Metabolomics

Whole-grain consumption is associated with a reduced risk of a number of diseases related to Western lifestyle such as obesity $^{(1,2)}, \mathrm{CVD}^{(3)}$ and type 2 diabetes $^{(4)}$. Whole grains contain cereal dietary fibres (DF) like arabinoxylan $(\mathrm{AX})$ and $(1 \rightarrow 3)$ $(1 \rightarrow 4)-\beta$-D-glucan (referred to as $\beta$-glucan; BG) that are known to attenuate postprandial blood glucose and insulin levels and lower serum cholesterol ${ }^{(5-8)}$; these are functionalities that have potential health beneficial effects.

Our knowledge of how DF regulates events at the molecular level is still limited and understanding the biochemical mechanisms behind the protective effects may lead to development of new health-promoting products with specifically designed fibre fractions. Combining nutriomic techniques like metabolomics with traditional measurements of biomarkers such as glucose and insulin levels may provide such information. High-DF/rye diets have shown to alter the concentrations of betaine and its related metabolites in the homocysteine metabolism in plasma of human subjects ${ }^{(9,10)}$ and animal models ${ }^{(11-14)}$, which may be associated with a reduced risk of CVD. Differences in plasma metabolites of branchedchain amino acids (BCAA), aromatic amino acids and lysophosphatidylcholine (lysoPC) species, together with some precursors and breakdown products, have furthermore been found in response to high-DF/rye diets that may mediate positive effects

\footnotetext{
Abbreviations: AX, arabinoxylan; BCAA, branched-chain amino acid; BG, $\beta$-glucan; $\triangle \mathrm{AV}$, portal-arterial difference; DF, dietary fibre; GR, dark ground rye; LC-MS, liquid chromatography-MS; lysoPC, lysophosphatidylcholine; PCA, principal components analysis; RK, rye kernels; WF, white wheat.
}

* Corresponding author: Dr Knud Erik Bach Knudsen, fax +45 87154249, email KnudErik.BachKnudsen@agrsci.dk 
related to reduced risks of obesity and diabetes ${ }^{(10,15,16)}$. In the above and other reported studies, the subjects had been adapted to the diets for at least $7 \mathrm{~d}$.

In the present study the porcine portal-arterial catheterisation model was used to study the acute metabolic response, similar to what is done when measuring glycaemic index in human subjects without any prior adaptation, to five test breads with different fibres and fibre levels. The test breads were a regular white wheat (WF) bread, two WF breads supplemented with either concentrated wheat AX or concentrated oat BG, a dark ground rye (GR) bread, and a rye bread with intact rye kernels (RK). Concentrated AX and BG have often proved their effects in comparison with low-fibre diets. It is, however, important to evaluate the clinical importance of concentrated DF relative to whole-grain DF, which has received little attention. As such, this design allowed for comparison of whole-grain/whole-kernel $v$. concentrated DF as well as a comparison of high fibre $v$. low fibre. The aim was to determine the properties of concentrated AX, BG, whole-grain rye and intact RK on glycaemic control using an untargeted liquid chromatography-MS (LC-MS) metabolomics approach.

\section{Experimental methods}

\section{Animal model}

A group of six female pigs (crossbreeds of Duroc $\times$ Danish Landrace $\times$ Yorkshire from the swineherd at Aarhus University, Foulum, Tjele, Denmark) with an initial body weight of $60 \cdot 2$ (SEM 3.1) kg were catheterised in the mesenteric artery and the portal vein using the method described by Jørgensen et al. ${ }^{(17)}$. Pigs were selected for surgery if they had a plasma glucose level below $5.8 \mathrm{mmol} / \mathrm{l}$ at $1 \mathrm{~h}$ after ingesting a traditional swine diet, and if they would eat rye bread during testing. The portal vein catheter was infused with 0.5 litres sterile saline on the first postoperative day to keep the fluid balance stable after surgery and keep the catheter patent and the pigs were exercised for $3 \mathrm{~d}$ postoperative. In total, 7-9 d were spent to recover. Catheters were flushed aseptically with $1000 \mathrm{IU} / \mathrm{ml}$ of heparin solution every 3-4 d and otherwise if needed to maintain patency. Catheters were secured using pouches attached to the side of the pig using Leucoplast/Tensoplast (BSN Medical) and an elastic tubular net. The pigs were kept individually in pens with a concrete floor. Elevated plastic grids covering half of the pen allowed the pigs to rest and stay dry.

The animal experiment was conducted according to the license obtained by the Danish Animal Experiments Inspectorate, Ministry of Food, Agriculture and Fisheries, Danish Veterinary and Food Administration.

\section{Breads}

The three commercial breads were produced at Lantmännen Schulstad A/S (Hammerholmen). Commercial names are: Sundbrød Hvede Toast (WF), Mørkt Rugbrød (GR) and Levebrød Multikernerugbrød (RK). The AX and BG breads were baked in-house. AX bread was made from white wheat flour $(678 \mathrm{~g} / \mathrm{kg})$, wheat AX concentrate $(244 \mathrm{~g} / \mathrm{kg}$; Manildra Group), water, baker's yeast $(35 \mathrm{~g} / \mathrm{kg})$, sugar $(9 \mathrm{~g} / \mathrm{kg})$, salt $(17 \mathrm{~g} / \mathrm{kg})$ and shortening $(18 \mathrm{~g} / \mathrm{kg})$. Soluble wheat AX was isolated from the soluble fraction after extraction of starch and gluten, concentrated by evaporation, heat treated, further treated with $\alpha$-amylase and glucoamylase, precipitated with ethanol $(1: 3, \mathrm{v} / \mathrm{v})$, filtered and finally dried on a spray dryer. BG was made from white wheat flour $(710 \mathrm{~g} / \mathrm{kg})$, Promoat ${ }^{\mathrm{TM}}$ $\left(133 \mathrm{~g} / \mathrm{kg} ;\right.$ Biovelop AB), Vitacel ${ }^{\circledR}$ WF $600 \quad(69 \mathrm{~g} / \mathrm{kg}$; Rettenmaier and Söhne $\mathrm{GmbH})$, wheat gluten $(10 \mathrm{~g} / \mathrm{kg})$, baker's yeast $(35 \mathrm{~g} / \mathrm{kg})$, sugar $(9 \mathrm{~g} / \mathrm{kg})$, salt $(17 \mathrm{~g} / \mathrm{kg})$ and shortening $(18 \mathrm{~g} / \mathrm{kg})$. Soluble BG was obtained from the sub-aleurone of oat by combining wet-milling and enzymic hydrolysis. Since a high dose of $\mathrm{BG}$ from Promoat ${ }^{\mathrm{TM}}$ would cause the dough to be very sticky, the amount was reduced and cellulose/purified wheat fibre $\left(\right.$ Vitacel $^{\circledR}$ ) was added to balance the DF content in the BG bread relative to the other high-DF breads. Details concerning molecular weight and molecular characteristics of the DF concentrates can be found in the papers of Kasprzak et al. ${ }^{(18,19)}$.

\section{Design}

Pigs were fed each of the five breads in a randomised $5 \times 6$ incomplete Latin square cross-over design. On sampling days the morning meal was replaced with experimental bread (Mondays and Thursdays, 3-4 d apart) that provided about $200 \mathrm{~g}$ of available carbohydrate and blood was collected in heparinised Vacutainers (Greiner Bio-One) from the mesenteric artery and portal vein. Plasma for metabolomics analysis was collected at -15 (fasting value, $\mathrm{t}_{0}$ ), 30, 60, 90, 120, 180 and $240 \mathrm{~min}$ postprandial. After collection, catheters were flushed with $5 \mathrm{ml} 0.9 \%$ sterile saline to replace fluid loss and filled with $100 \mathrm{IU} / \mathrm{ml}$ heparin solution to prevent clotting. Packed cell volume values were measured at -15 and 240 min. Blood was centrifuged at $2000 \mathrm{~g}$ for $12 \mathrm{~min}$ at $4^{\circ} \mathrm{C}$ and plasma was kept frozen at $-80^{\circ} \mathrm{C}$ until further analysis. Between interventions the pigs were fed a washout diet low in DF consisting of wheat flour $(807 \mathrm{~g} / \mathrm{kg})$, Lacprodan ${ }^{\circledR}-87$ (73 g/kg; Arla Foods Ingredients), rapeseed oil (30 g/ kg), Vitacel $^{\circledR}$ WF 600 (Rettenmaier and Söhne $\mathrm{GmbH}$ ) and a vita$\mathrm{min} /$ mineral mixture containing synthetic amino acids providing all the necessary vitamins and minerals. Pigs were fed three times per $\mathrm{d}$ with $635 \mathrm{~g}$ of washout diet per meal. When reaching $70 \mathrm{~kg}$ of body weight the dose was $750 \mathrm{~g}$. Once per week, the pigs were weighed and received an intramuscular supplement of $400 \mathrm{mg} \mathrm{Fe}^{3+}$ (Uniferon ${ }^{\circledR}$; Pharmacosmos A/S). The pigs had free access to water during the entire study period.

\section{Analytical methods}

Test breads and the washout diet were freeze-dried and ground to a particle size less than $0.5 \mathrm{~mm}$ for chemical analyses. All analyses were performed in duplicate. DM content was determined by oven drying at $103^{\circ} \mathrm{C}$ for $20 \mathrm{~h}$. Gross energy was analysed by use of an oxygen bomb calorimeter (Parr Instrument Company). Ash was analysed by an AOAC method $^{(20)}$. Protein $(\mathrm{N} \times 6 \cdot 25)$ was measured by Dumas ${ }^{(21)}$. 
Amino acids were quantified after hydrolysis for $23 \mathrm{~h}$ at $110^{\circ} \mathrm{C}$ with or without performic acid oxidation using ion exchange chromatography and photometric detection after ninhydrin reaction $^{(22)}$. Fat was extracted with diethyl ether after $\mathrm{HCl}$ hydrolysis according to the Stoldt procedure ${ }^{(23)}$. Starch and NSP were analysed essentially as described by Bach Knudsen ${ }^{(24)} ; 2 \mathrm{M}^{-} \mathrm{H}_{2} \mathrm{SO}_{4}$ for $1 \mathrm{~h}$ was used instead of 1 $\mathrm{M}-\mathrm{H}_{2} \mathrm{SO}_{4}$ for $2 \mathrm{~h}$ for the NSP analysis. The content of nondigestible carbohydrates (NDC) was determined by direct acid hydrolysis without starch removal and alcohol precipitation. The total NDC was calculated by subtraction of the starch content. AX was calculated as the sum of arabinose and xylose from the NSP analysis. BG was analysed by the enzymic-colorimetric method of McCleary \& GlennieHolmes ${ }^{(25)}$. Klason lignin was measured gravimetrically as the sulfuric acid-insoluble residue as described by Theander $\& \AA \operatorname{man}^{(26)}$. The content of available/digestible carbohydrates was calculated as:

Available carbohydrates $=$ starch + fructose + sucrose

Chemical compositions of the test breads are presented in Table 1.

Plasma insulin was analysed by time-resolved fluoroimmunoassay as described by Løvendahl \& Purup ${ }^{(27)}$ and the insulinaemic indices were calculated from the 120 min incremental area under the insulin curves using the WF bread as the reference.

\section{Reversed-phase HPLC-MS}

Plasma samples $(200 \mu \mathrm{l})$ were mixed with $20 \mu \mathrm{l} 100 \mu \mathrm{g} / \mathrm{ml}$ internal standard mix of glycocholic acid (glycine- $1-{ }^{13} \mathrm{C}$ ) and

Table 1. Chemical composition, amount of available carbohydrate $(\mathrm{CHO})$ per meal and insulinaemic index (II, 120 min, using the white wheat (WF) bread as the reference) of the experimental test breads

\begin{tabular}{lrrrrr}
\hline & \multicolumn{5}{c}{ Bread } \\
\cline { 2 - 6 } Chemical composition & WF & AX & BG & GR & RK \\
(g/kg DM) & 634 & 708 & 615 & 520 & 543 \\
\hline DM (g/kg as-fed basis) & 18 & 19 & 18 & 18 & 18 \\
Gross energy (MJ/kg DM) & 24 & 42 & 29 & 36 & 32 \\
Ash & 132 & 210 & 122 & 95 & 91 \\
Protein (N ×6.25) & 8 & 12 & 7 & 5 & 5 \\
$\quad$ Leucine & 5 & 9 & 5 & 4 & 4 \\
Valine & 3 & 6 & 3 & 2 & 2 \\
$\quad$ Tyrosine & 1 & 2 & 1 & 1 & 1 \\
$\quad$ Tryptophan & 6 & 8 & 5 & 4 & 4 \\
Phenylalanine & 34 & 28 & 31 & 22 & 23 \\
Fat & 711 & 514 & 612 & 588 & 608 \\
Starch & 3 & 3 & 52 & 21 & 19 \\
BG & 17 & 78 & 32 & 76 & 77 \\
AX & 77 & 212 & 199 & 209 & 220 \\
Dietary fibre (NDC + lignin) & 210 & 225 & 229 & 210 & 211 \\
Available CHO (g/meal) & & & & & \\
II (\%) & 100 & 93 & 79 & 61 & 78 \\
$\quad$ Mean & & 17 & 17 & 17 & 17 \\
SD & &
\end{tabular}

AX, arabinoxylan; BG, $\beta$-glucan; GR, dark ground rye; RK, rye kernels; NDC, nondigestible carbohydrates.
lysoPC (17:0). The samples were added to $600 \mu$ ice-cold methanol, immediately vortexed and incubated at $4{ }^{\circ} \mathrm{C}$ for $20 \mathrm{~min}$ for deproteinisation. The supernatant fractions were collected after centrifugation at $13200 \mathrm{~g}$ for $10 \mathrm{~min}$ and evaporated to dryness under a stream of $\mathrm{N}_{2}$. The resulting dry residues were re-suspended in $200 \mu \mathrm{l}$ water-acetonitrileformic acid (95:5:0.1, by vol.) and centrifuged at $10621 \mathrm{~g}$ for $10 \mathrm{~min}$. The supernatant fractions were injected into the LC-MS.

For the LC-MS analysis, an Ultimate 3000 (Dionex) HPLC system was coupled to a MicrOTOF-Q II mass spectrometer (Bruker Daltonik $\mathrm{GmbH}$ ) operating in positive electrospray ionisation mode. The scan range was from 50 to $1000 \mathrm{~m} / \mathrm{z}$ at a sampling rate of $2 \mathrm{~Hz}$. The capillary voltage was 4500 $\mathrm{V}$, the nebulising gas pressure was $1.8 \mathrm{bar}$ and the drying gas flow and temperature were 8.0 litres $/ \mathrm{min}$ and $200^{\circ} \mathrm{C}$, respectively. Lithium formate at a concentration of $5 \mathrm{~mm}$ in water-isopropanol-formic acid (50:50:0.2, by vol.) was employed as an external calibrant. For MS/MS analysis, Ar was used as the collision gas, and collisions were carried out at energies from 6 to $60 \mathrm{eV}$. All other parameters were the same as above.

Chromatographic separation was performed on a Discovery ${ }^{B}$ HS $\mathrm{C}_{18}$ column $(15 \mathrm{~cm} \times 2 \cdot 1 \mathrm{~mm}, 3 \mu \mathrm{m})$ used together with a $\mathrm{C}_{18}$ pre-column. The column was held at $30^{\circ} \mathrm{C}$. The injection volume was $5 \mu \mathrm{l}$. Mobile phase A consisted of water-formic acid $(100: 0 \cdot 1, \mathrm{v} / \mathrm{v})$ and mobile phase B consisted of acetonitrile-formic acid $(100: 0 \cdot 1, \mathrm{v} / \mathrm{v})$. The column was equilibrated for 5 min before the gradient started at $10 \% \mathrm{~B}$ for $1 \mathrm{~min}$ and then linearly increased to $100 \% \mathrm{~B}$ within $20 \mathrm{~min}$. The mobile phase was kept at isocratic conditions $(100 \% \mathrm{~B})$ for $5 \mathrm{~min}$ and then turned back to $10 \% \mathrm{~B}$. Total analysis time was $31 \mathrm{~min}$ and the flow rate was $200 \mu \mathrm{l} / \mathrm{min}$. A sample of pooled plasma and blanks was re-injected after each seven samples for quality control.

\section{Data analysis}

Acquired mass spectra were calibrated and converted into mzXML file format using CompassXport (Bruker Daltonik $\mathrm{GmbH})$ to carry out data analysis. MZmine $2.3^{(28)}$ was employed for pre-processing of data using a centroid peak detector algorithm and the RANSAC aligner. A matrix was generated with retention time, $\mathrm{m} / \mathrm{z}$ and respective intensities of ions. Data were explored as arterial and portal vein samples, and as portal-arterial differences $(\Delta \mathrm{AV})$. Arterial and portal vein data remained non-normalised, whereas the $\Delta \mathrm{AV}$ were calculated from normalised data, so that arterial intensities of each metabolite could be subtracted from the venous intensities. Normalisation was performed using the standard compound normaliser in MZmine 2.3 with a weighted contribution of all internal standards. Orthogonal signal correction according to Wold $e t$ al. ${ }^{(29)}$ was applied using the PLS toolbox for MATLAB 7.11.0 (R2010b) (The MathWorks, Inc.) to selectively remove irrelevant variation between pigs. Data were pareto scaled and principal components analysis (PCA) was performed using LatentiX 2.00 (Latent5 Aps). Outliers were removed based on $95 \%$ CI and plots of residual 
variance $v$. Hotelling's $\mathrm{T}^{2}$ after which the models were recalculated. Loading plots were used to detect metabolite ions with the greatest influence on clustering. Compounds were identified based on database searches in METLIN (http://metlin. scrips.edu), The Human Metabolome Database (http:// www.hmdb.ca) and PubChem compound database (http:// pubchem.ncbi.nlm.nih.gov) using accurate mass and mass spectrometric fragmentation patterns. Authentic standards were used for confirmation of the metabolites when available.

Effects of breads, time and their interactions as well as sampling site (artery or vein) were analysed as repeated measurements using the MIXED procedure of SAS (SAS, version 9.3; SAS Institute Inc.). Compounds were analysed as a linear mixed model:

$$
Y_{i j k l}=\mu+\alpha_{i}+\beta_{j}+\alpha \beta_{i j}+\gamma_{k}+\delta_{i k l}+\rho_{i j k l}+\varepsilon_{i j k l},
$$

where $Y_{i j k l}$ is the dependent variable, $\mu$ is the overall mean, $\alpha_{i}$ is the effect of bread ( $i=$ WF, GR, RK, BG, AX), $\beta_{j}$ is the time after feeding $(j=-15(0), 15,30,45,60,90,120$, 180 and 240), and $\alpha \beta_{i j}$ is the interaction term. The three terms $y_{\mathrm{k}}(k=$ pig $1, \ldots),, \quad \delta_{i k l}(l=$ period $1, \ldots, 5)$ and $\rho_{i j k l}$ accounted for repeated measurements being performed on the same pig $\left(y_{k}\right)$ among periods in the cross-over design $\left(\boldsymbol{\delta}_{i k l}\right)$, and on the same pig within a sampling day $\left(\rho_{i j k l}\right)$. $\boldsymbol{\varepsilon}_{i j k l}$ is the residual error component. The covariance structure of $\rho_{i j k l}$ was modelled using the spatial power option, which takes into account the different intervals between repeated measurements. Pearson correlation coefficients were calculated using the CORR procedure of SAS. The correlation of glucose and leucine to insulin was further analysed by a multiple regression correlation analysis using the REG procedure of SAS. If variance homogeneity was not present the data were $\ln (x)$ transformed before statistical analysis. Statistical significance was set as $P<0.05$ and $0.05 \leq P$ $<0 \cdot 10$ as trends.

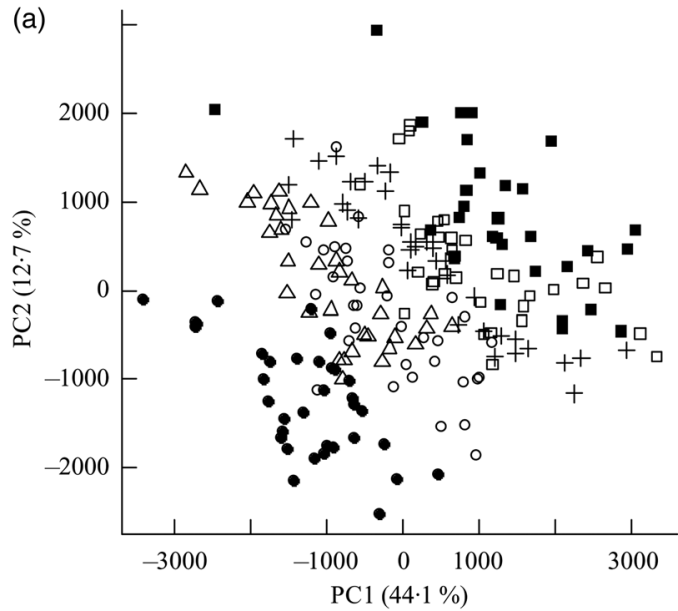

\section{Results}

Orthogonal signal correction-filtered PCA models of arterial samples individually and portal vein samples individually showed similar separation patterns according to intake of the different bread types (Fig. 1). The separations were caused by glucose and amino acids. Significantly higher glucose concentrations were found for the WF bread compared with the AX, GR and RK breads in the portal vein but not in the artery (Table 2). Plasma levels of tyrosine, leucine, phenylalanine and tryptophan were increased in response to the AX bread and decreased in response to the rye breads compared with WF bread. This clearly mirrored the protein and amino acid compositions in the breads, with significant correlations between breads and plasma (Table 2). A correlation between the insulin response and glucose and leucine was also found (Table 2). A correlation between insulin and the other amino acids was not detected. Leucine, however, might influence insulin due to its correlation with glucose uptake $(P<0.001)$, which is why a multiple regression correlation analysis was performed. The isolated effects of leucine were found to affect insulin in the mesenteric artery $(P=0.001)$ but not in the portal vein $(P=$ $0.41)$, whereas the effects of glucose on insulin was significant in both the artery and vein $(P<0.001)$.

The $\triangle \mathrm{AV}$ were calculated and reflected the net absorption of metabolites from the gastrointestinal tract. The orthogonal signal correction-calibrated PCA model revealed a clear distinction and variation in metabolite absorption due to bread type on principal component 1 (Fig. 2). The two rye breads, with almost similar chemical compositions apart from the milled/intact kernels, were grouped very distantly and RK bread showed a greater resemblance to the AX bread than the GR bread.

Metabolites with greatest influence on the clustering were located (Table 3). However, large variations in the $\Delta \mathrm{AV}$ were found due to the semi-quantitative nature of the data, which caused very few significant bread differences and no clear correlations between plasma and bread contents or insulin. Time profiles of metabolite compounds of $\Delta \mathrm{AV}$ of

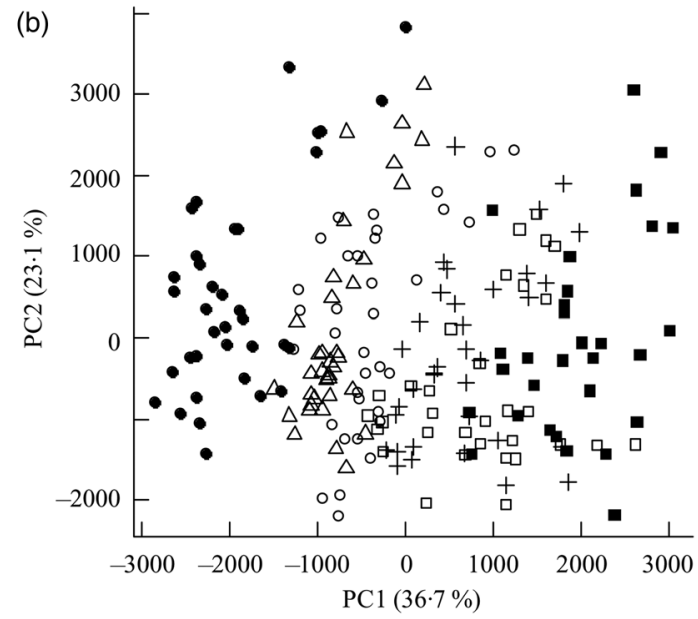

Fig. 1. Principal components analysis scores plot of plasma from the mesenteric artery (a) and the portal vein (b) of pigs taken at $0,30,60,90,120,180$ and 240 min after consumption of five different test breads. $\mathbf{\square}$, Fasting values; $\Delta$, white wheat bread; $\bullet$, arabinoxylan bread; $\bigcirc, \beta$-glucan bread; + , dark ground rye bread; $\square$, rye bread with kernels. The amount of total variation accounted for by the principal components PC1 and PC2 is shown in parentheses. 


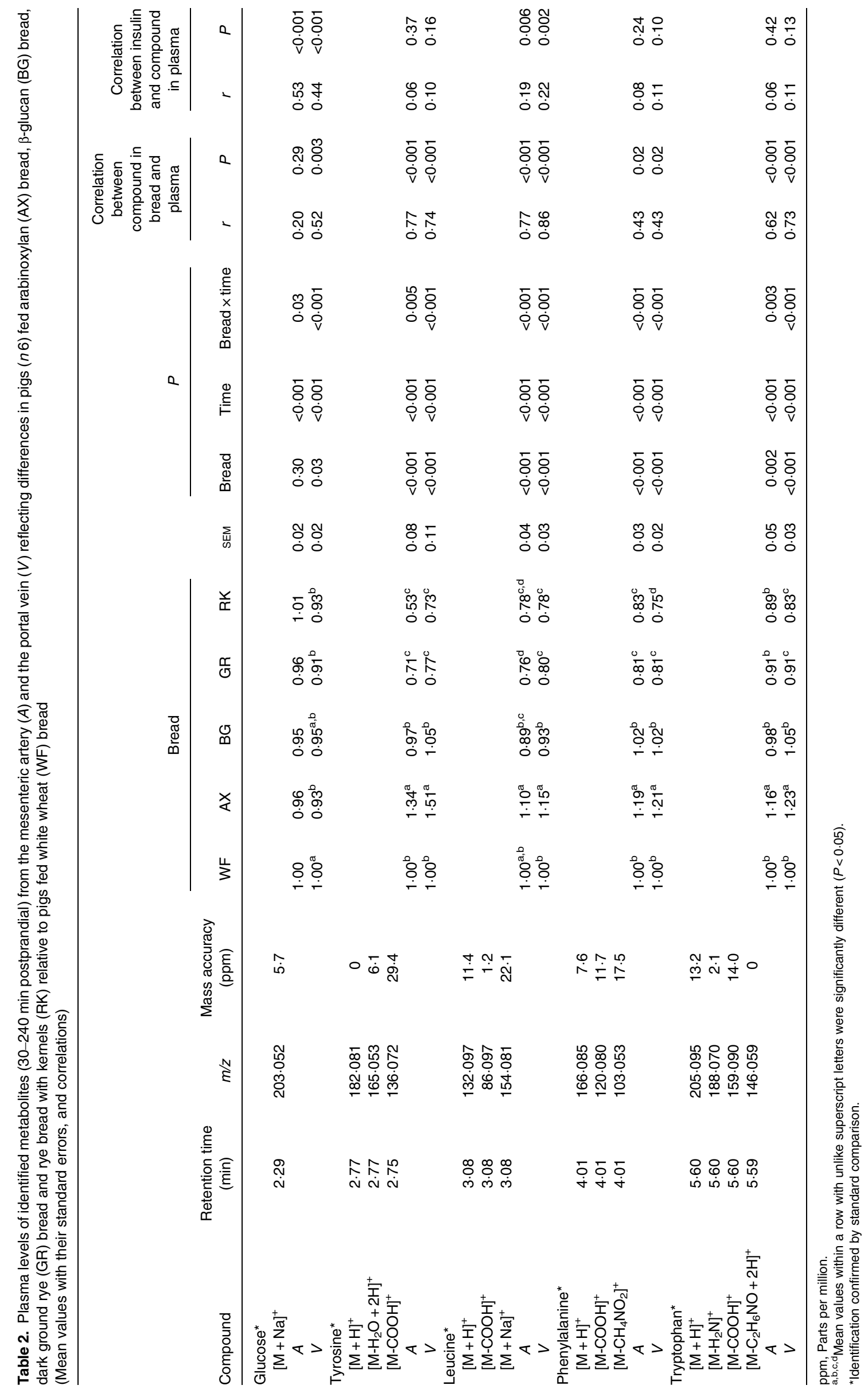




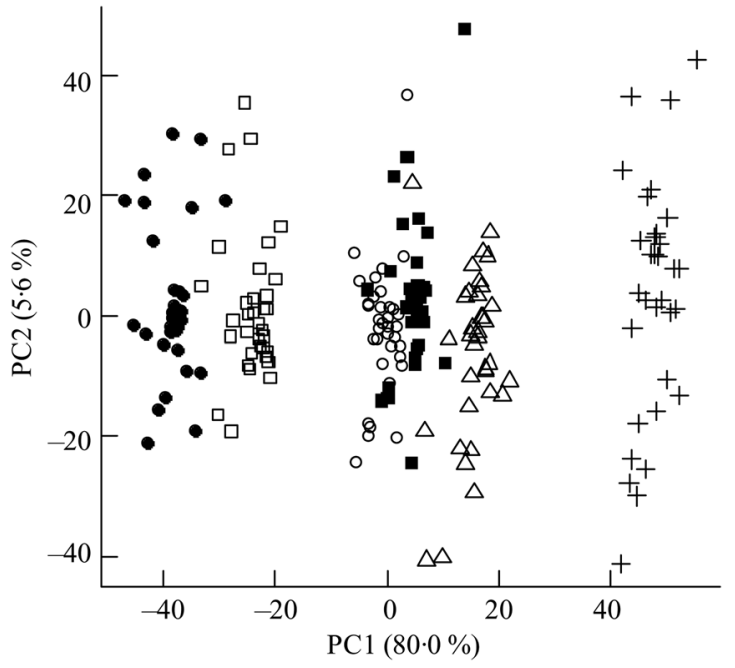

Fig. 2. Principal components analysis scores plot of portal-arterial differences in pigs fed five different test breads. Plasma was sampled at $0,30,60,90,120$, 180 and 240 min postprandial. $\mathbf{\square}$, Fasting values; $\Delta$, white wheat bread; $\boldsymbol{\bullet}$, arabinoxylan bread; $\bigcirc, \beta$-glucan bread; + , dark ground rye bread; $\square$, rye bread with kernels. The amount of total variation accounted for by the principal components PC1 and PC2 is shown in parentheses.

glucose, leucine, phenylalanine and lysoPC 18:2 are depicted in Fig. 3. The WF bread had a numerically higher glucose peak than the AX, GR and RK breads, although not significant (Fig. 3(a)) and leucine absorption was numerically higher for $\mathrm{AX}$ and BG breads than the other breads (Fig. 3(b)). Absorption of phenylalanine (Fig. 3(c)) and tryptophan (data not shown) was significantly lower for the RK bread compared with the WF-based breads (WF, AX and BG breads). WF, $B G$ and GR breads tended to induce increased levels of lysoPC 18:2, postprandial, whereas AX and RK breads maintained baseline levels or slight decreases (Fig. 3(d)) - similarly for lysoPC 20:4 and 16:0 (data not shown).

\section{Discussion}

Studies in human subjects only allow collection of peripheral blood in which many metabolites have been metabolised by the liver and other tissues. Consequently, several effects cannot be measured. The porcine portal-arterial catheterisation model allows simultaneous blood draws from the mesenteric artery and the portal vein; portal blood permits measurements of absorbed nutrients and gut hormones from the intestine to the liver, whereas arterial blood represents the systemic circulation. The $\triangle \mathrm{AV}$ thus represents the enrichment of compounds coming from the gastrointestinal tract ${ }^{(30)}$. In the present study the acute metabolic effects to five breads were studied in plasma from the porcine portal-arterial catheterisation model. LC-MS metabolomics was applied to gain additional insight into the effects of $A X$ and $B G$, whole-grain rye and refined white wheat.

The four high-fibre breads had 61-65\% more DF than the WF bread; for AX, GR and RK breads predominantly in form of $\mathrm{AX}$ and for BG bread in form of BG. The different breads also incurred significant differences in the physico-chemical properties of digesta ${ }^{(19)}$. This had an impact on glucose absorption, showing glucose to be attenuated in response to the AX, GR and RK breads. Decreased glucose absorption could be due to delayed gastric emptying and an increased viscosity of digesta caused by the high AX contents as seen in an accompanying study with ileal-cannulated pigs fed the same bread diets by Kasprzak et al. ${ }^{(19)}$. Increased viscosity decreases availability of digestive enzymes to the food bolus, thereby reducing glucose absorption. A reduced glucose uptake causes an attenuated insulin response that may prevent the development of diseases related to the metabolic syndrome. The BG bread was not found to reduce glucose absorption due to degradation of the $B G$ content in the small intestine of the pigs, which was found in the study with ileal-cannulated pigs ${ }^{(19)}$. Glycaemic index measurements from human subjects with the metabolic syndrome ${ }^{(31)}$ and quantitative glucose measurements from catheterised pigs ${ }^{(32)}$ have shown similar reductions in glucose absorption but only significant for the AX bread. The LC-MS data were only semi-quantitative, but the data seemed to mimic the quantitative results, which may validate the metabolomics method.

Plasma levels in the portal vein and the mesenteric artery correlated with the amino acid contents in the breads, showing uptake and concentration to be linked. The AX bread had a high protein content originating from the refined wheat $\mathrm{AX}$ concentrate, which explains why AX bread yielded the highest content of amino acids in plasma. Protein levels between the breads, however, also significantly affected the insulin secretion through leucine. The ability of the liver to metabolise BCAA is limited ${ }^{(33)}$, which could explain why the effects of leucine were evident in the artery but not the portal vein. BCAA, especially leucine, are known to influence insulin responses ${ }^{(34,35)}$. Increased plasma levels of BCAA and phenylalanine have furthermore been associated with an increased risk of diabetes ${ }^{(36)}$. Two other studies with human subjects found leucine and isoleucine to be decreased after 8 or 12 weeks intervention with rye compared with oat and wheat $^{(10,16)}$. However, consumption of other food products and differences in protein intake could affect the results in these studies. On the other hand, rye may have a positive effect on BCAA metabolism to lower plasma levels that could be linked to decreased insulin secretion ${ }^{(10)}$; several studies report rye to lower postprandial insulin responses without affecting glycaemia ${ }^{(37-39)}$. Either way, these results warrant a more careful consideration concerning protein contents when investigating carbohydrate metabolism.

The lysoPC species were very dominant from the PCA models of $\triangle \mathrm{AV}$; the bread effects found were, however, minor. LysoPC are major plasma lipid components that serve as signalling molecules and as transporters of fatty acids, phosphatidylglycerol and choline ${ }^{(40)}$, but different lysoPC species may have different functions and may exert different effects. A study with human subjects found increased concentrations of lysoPC during an oral glucose tolerance test ${ }^{(41)}$. Another study found higher plasma contents of lysoPC after an intervention with wheat, oat and potato compared with rye ${ }^{(16)}$. This indicates that glucose uptake changes the lipid profile of lysoPC, whereas DF or other bioactive/antioxidant compounds in rye might counteract the effect. Studies investigating 


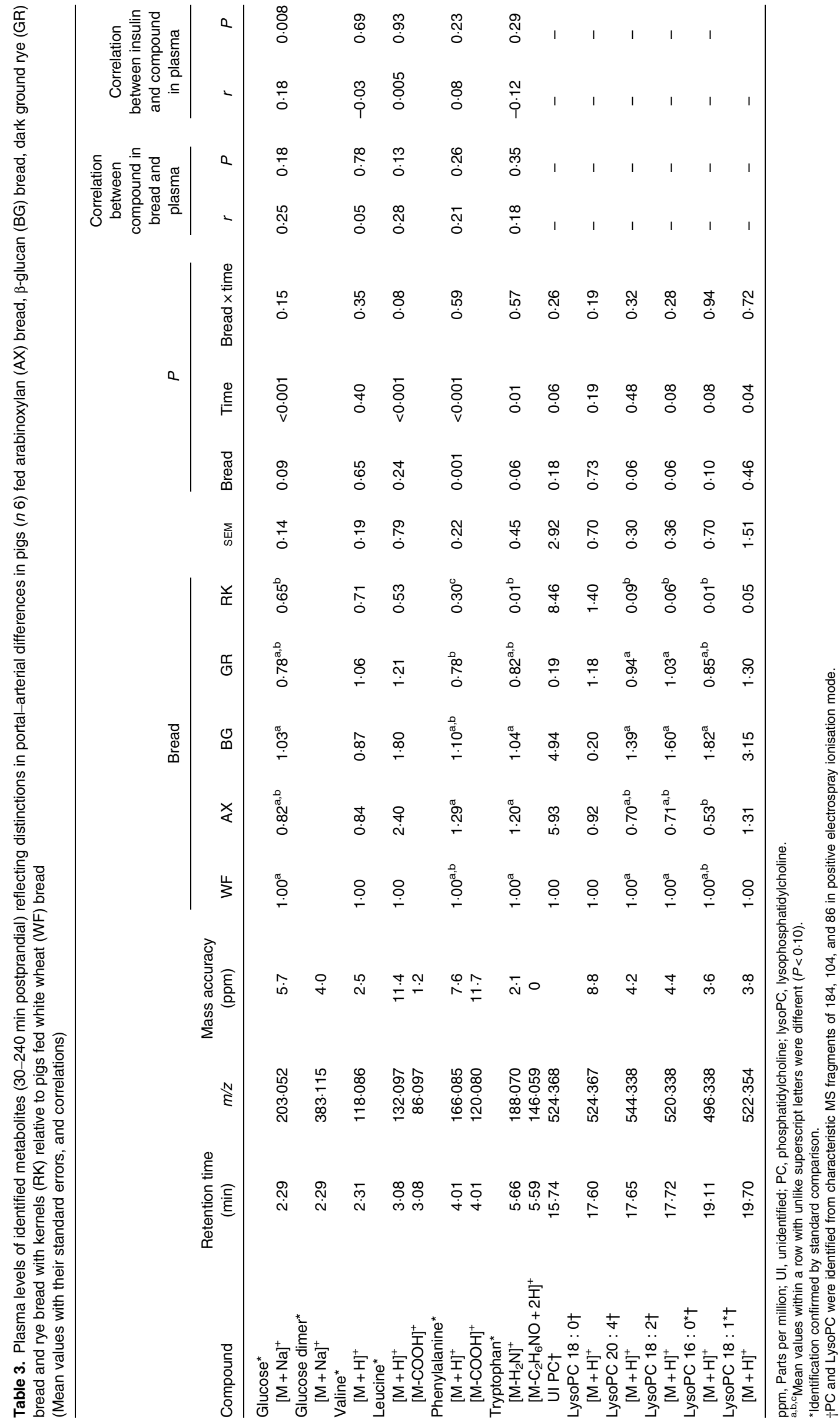



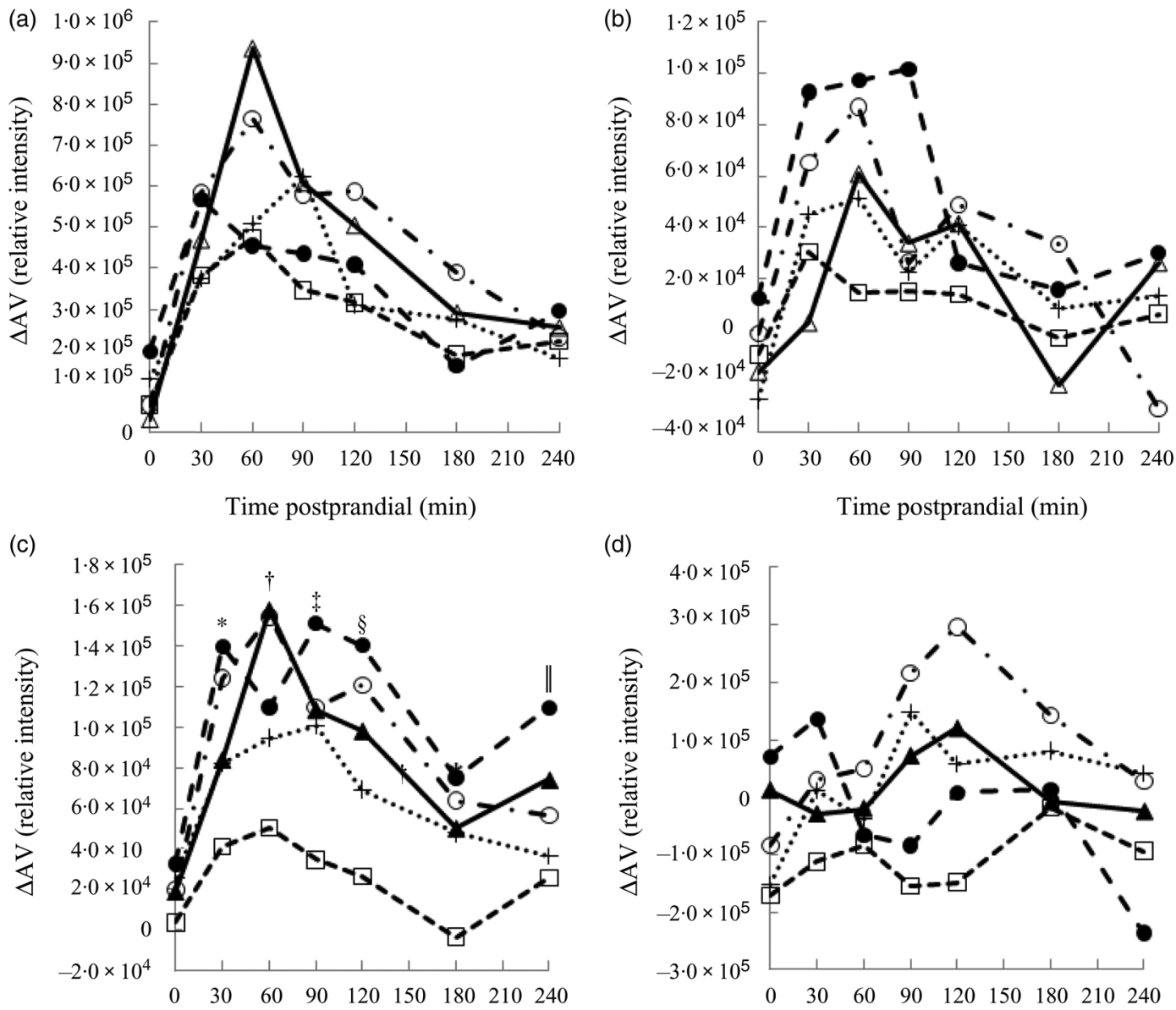

(d)

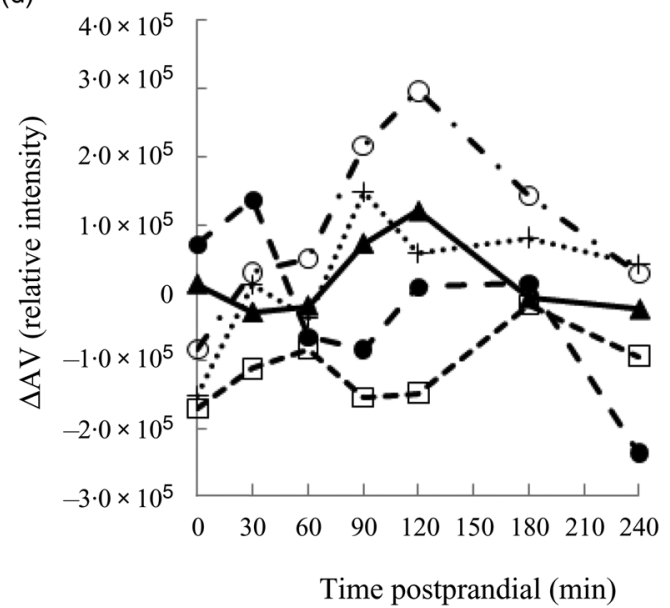

Fig. 3. Time profiles of identified plasma metabolites in pigs fed five different test breads: (a) glucose (pooled SEM $\left.=8.0 \times 10^{4}\right) ;\left(\right.$ b) leucine (pooled SEM $\left.=1.7 \times 10^{4}\right) ;(\mathrm{c})$ phenylalanine (pooled SEM $\left.=4.1 \times 10^{4}\right)$; (d) lysophosphatidylcholine $18: 2$ (pooled SEM $\left.=1.1 \times 10^{5}\right)$. 4 , White wheat bread $(W F)$; $\bullet$, arabinoxylan bread $(A X)$; $O$, $\beta$-glucan bread (BG); + , dark ground rye bread (GR); $\square$, rye bread with kernels (RK). Means at the same time point differ: ${ }^{*} \mathrm{RK}<\mathrm{AX}, \mathrm{BG}(P<0.05)$; $\dagger \mathrm{RK}<\mathrm{BG}$, WF $(P<0.003)$; ‡ RK $<\mathrm{AX}, \mathrm{BG}, \mathrm{GR}, \mathrm{WF}(P<0.05)$; § GR $<\mathrm{AX}$ and RK $<\mathrm{AX}, \mathrm{BG}, \mathrm{WF}(P<0.03)$; $\| \mathrm{GR}, \mathrm{RK}<\mathrm{AX}(P<0.03)$. $\Delta \mathrm{AV}$, portal-arterial difference.

acquired obesity have additionally found lysoPC levels in plasma to be increased ${ }^{(42,43)}$ and they are major plasma lipid components of oxidised LDL with implications in atherosclerosis and chronic inflammation ${ }^{(40,44)}$. Therefore, decreased concentrations may be of interest to reduce the risk of lifestyle diseases. In this case WF, BG and GR breads tended to cause higher concentrations of lysoPC 18:2, 20:4 and 16:0 in plasma of the pigs than AX bread and especially RK bread. The explanation might be due to an attenuated glucose uptake with AX and RK breads. However, this was also true for GR bread. The reason for this difference between GR $v$. RK and AX breads is unknown.

In summary, analysis of the plasma metabolome of portalarterial catheterised pigs revealed differences in the acute response related to DF and protein contents of the breads. Detection of more DF-related markers was lacking and needs to be optimised in future studies. However, due to the short time span of plasma collection for $4 \mathrm{~h}$ postprandial, microbial degradation products, nutritive and non-nutritive (phytochemicals), could not be found in the present study. On the other hand, AX, GR and RK breads were found to efficiently decrease glucose absorption compared with WF bread, most probably due to the high AX contents. This indicates that concentrated AX incorporated into WF bread has similar effects on the glycaemic response as AX present in whole-grain rye bread, and may be useful in the prevention and treatment of obesity and diabetes. Decreased levels of some lysoPC species in response to AX and RK breads further indicated their beneficial effects on metabolism. Detection of BCAA from the breads to influence insulin secretion warrants a closer consideration regarding protein contents in intervention studies concerning carbohydrate metabolism.

\section{Acknowledgements}

The authors thank Winnie Østergaard Thomsen, Lisbeth Märcher, Karin Eybye, Lars Bilde Gildbjerg, Natalja Pustovalova Nørskov and the staff at the institute's animal facility for valuable technical assistance. Lantmännen Schulstad A/S is acknowledged for providing the rye breads and the WF bread, and BioVelop $\mathrm{AB}$ for providing the PromOat ${ }^{\circledR}$.

The BioFunCarb (Biofunctional Carbohydrates) project was financially supported by The Danish Council for Strategic 
Research (project no. 2101-08-0068) and the Nordic Centre of Excellence SYSDIET (project no. 070014).

K. L. N., M. S. H., H. N. L., H. J. and K. E. B. K. designed the research; K. L. N., M. S. H. and H. N. L. conducted the research; K. L. N. analysed data and wrote the first draft of the paper. All authors contributed, commented and approved the final content.

There are no conflicts of interest to declare.

\section{References}

1. Smith CE \& Tucker KL (2011) Health benefits of cereal fibre: a review of clinical trials. Nutr Res Rev 24, 118-131.

2. Liu SM, Willett WC, Manson JE, et al. (2003) Relation between changes in intakes of dietary fiber and grain products and changes in weight and development of obesity among middle-aged women. Am J Clin Nutr 78, 920-927.

3. Lairon D, Arnault N, Bertrais S, et al. (2005) Dietary fiber intake and risk factors for cardiovascular disease in French adults. $A m \mathrm{~J}$ Clin Nutr 82, 1185-1194.

4. Montonen J, Knekt P, Jarvinen R, et al. (2003) Whole-grain and fiber intake and the incidence of type 2 diabetes. Am J Clin Nutr 77, 622-629.

5. Wood PJ (2010) Oat and rye $\beta$-glucan: properties and function. Cereal Chem 87, 315-330.

6. Bach Knudsen KE \& Lærke HN (2010) Rye arabinoxylans: molecular structure, physicochemical properties and physiological effects in the gastrointestinal tract. Cereal Chem 87, 353-362.

7. Othman RA, Moghadasian MH \& Jones PJH (2011) Cholesterol-lowering effects of oat $\beta$-glucan. Nutr Rev 69, 299-309.

8. Gemen R, de Vries JF \& Slavin JL (2011) Relationship between molecular structure of cereal dietary fiber and health effects: focus on glucose/insulin response and gut health. Nutr Rev 69, 22-33.

9. Moazzami AA, Zhang JX, Kamal-Eldin A, et al. (2011) Nuclear magnetic resonance-based metabolomics enable detection of the effects of a whole grain rye and rye bran diet on the metabolic profile of plasma in prostate cancer patients. J Nutr 141, 2126-2132.

10. Moazzami AA, Bondia-Pons I, Hanhineva K, et al. (2012) Metabolomics reveals the metabolic shifts following an intervention with rye bread in postmenopausal women- a randomized control trial. Nutr J 11, 88.

11. Bertram HC, Bach Knudsen KE, Serena A, et al. (2006) NMR-based metabonomic studies reveal changes in the biochemical profile of plasma and urine from pigs fed high-fibre rye bread. Br J Nutr 95, 955-962.

12. Bertram HC, Malmendal A, Nielsen NC, et al. (2009) NMR-based metabonomics reveals that plasma betaine increases upon intake of high-fiber rye buns in hypercholesterolemic pigs. Mol Nutr Food Res 53, 1055-1062.

13. Fardet A, Canlet C, Gottardi G, et al. (2007) Whole-grain and refined wheat flours show distinct metabolic profiles in rats as assessed by a H-1 NMR-based metabonomic approach. $J$ Nutr 137, 923-929.

14. Yde CC, Bertram HC \& Bach Knudsen KE (2010) NMR-based metabonomics reveals distinct metabolic profiles of plasma from sows after consumption of diets with contrasting dietary fibre levels and composition. Livest Sci 133, 26-29.

15. Lankinen M, Schwab U, Seppänen-Laakso T, et al. (2011) Metabolomic analysis of plasma metabolites that may mediate effects of rye bread on satiety and weight maintenance in postmenopausal women. $J$ Nutr 141, 31-36.

16. Lankinen M, Schwab U, Gopalacharyulu PV, et al. (2010) Dietary carbohydrate modification alters serum metabolic profiles in individuals with the metabolic syndrome. Nutr Metab Cardiovasc Dis 20, 249-257.
17. Jørgensen H, Serena A, Theil PK, et al. (2010) Surgical techniques for quantitative nutrient digestion and absorption studies in the pig. Livest Sci 133, 57-60.

18. Kasprzak MM, Laerke HN \& Bach Knudsen KE (2012) Changes in molecular characteristics of cereal carbohydrates after processing and digestion. Int J Mol Sci 13, 16833-16852.

19. Kasprzak MM, Lærke HN \& Bach Knudsen KE (2012) Effects of isolated and complex dietary fiber matrices in breads on carbohydrate digestibility and physicochemical properties of ileal effluent from pigs. J Agric Food Chem 60, 12469-12476.

20. AOAC (1990) Official Methods of Analysis, 15th ed. Arlington, VA: AOAC.

21. Hansen B (1989) Determination of nitrogen as elementary-N, an alternative to Kjeldahl. Acta Agric Scand 39, 113-138.

22. European Commission (1998) Commission Directive 98/64/EC of 3 September 1998 Establishing Community Methods of Analysis for the Determination of Amino-Acids, Crude Oils and Fats, and Olaquindox in Feedingstuffs and Amending Directive 71/393/EEC. Brussels: The Commission of the European Communities.

23. Stoldt W (1952) Vorschlag zur Vereinheitlichung der Fettbestimmung in Lebensmitteln (Proposal to standardise the determination of fat in food). F Seif 54, 206-207.

24. Bach Knudsen KE (1997) Carbohydrate and lignin contents of plant materials used in animal feeding. Anim Feed Sci Tech 67, 319-338.

25. McCleary BV \& Glennie-Holmes MJ (1985) Enzymatic quantification of $(1 \rightarrow 3)(1 \rightarrow 4)-\beta$-D-glucan in barley and malt. J Inst Brew $\mathbf{9 1}$, 285-295.

26. Theander O \& Åman P (1979) Studies on dietary fibers. 1. Analysis and chemical characterization of water-soluble and water-insoluble dietary fibers. Swed J Agr Res 9, 97-106.

27. Løvendahl P \& Purup HM (2002) Technical note: time-resolved fluoro-immunometric assay for intact insulin in livestock species. J Anim Sci 80, 191-195.

28. Pluskal T, Castillo S, Villar-Briones A, et al. (2010) MZmine 2: modular framework for processing, visualizing, and analyzing mass spectrometry-based molecular profile data. BMC Bioinformatics 11, 395.

29. Wold S, Antti H, Lindgren F, et al. (1998) Orthogonal signal correction of near-infrared spectra. Chemometr Intell Lab Syst 44, 175-185.

30. Rerat AA (1980) Some quantitative aspects of protein and carbohydrate absorption in the pig. Proc Nutr Soc 39, 177-184.

31. Hartvigsen ML, Gregersen S, Lærke HN, et al. (2014) Effects of concentrated arabinoxylan and $\beta$-glucan compared with refined wheat and whole grain rye on glucose and appetite in subjects with the metabolic syndrome: a randomized study. Eur J Clin Nutr 68, 84-90.

32. Christensen KL, Hedemann MS, Lærke HN, et al. (2013) Concentrated arabinoxylan but not concentrated $\beta$-glucan in wheat bread has similar effects on postprandial insulin as wholegrain rye in porto-arterial catheterized pigs. J Agric Food Chem 61, $7760-7768$

33. Leverve XM (2003) Integration of metabolism 2: protein and amino acids. In Nutrition and Metabolism, pp. 43-73 [MJ Gibney, IA Macdonald and HM Roche, editors]. Oxford: Blackwell Publishing.

34. Floyd JC, Fajans SS, Conn JW, et al. (1966) Insulin secretion in response to protein ingestion. J Clin Invest 45, 1479-1486.

35. Nilsson M, Holst JJ \& Björck IME (2007) Metabolic effects of amino acid mixtures and whey protein in healthy subjects: studies using glucose-equivalent drinks. Am J Clin Nutr 85, 996-1004.

36. Wang TJ, Larson MG, Vasan RS, et al. (2011) Metabolite profiles and the risk of developing diabetes. Nat Med 17, 448-453.

37. Leinonen K, Liukkonen K, Poutanen K, et al. (1999) Rye bread decreases postprandial insulin response but does not alter glucose response in healthy Finnish subjects. Eur J Clin Nutr 53, 262-267.

38. Juntunen KS, Niskanen LK, Liukkonen KH, et al. (2002) Postprandial glucose, insulin, and incretin responses to grain products in healthy subjects. Am J Clin Nutr 75, 254-262. 
39. Juntunen KS, Laaksonen DE, Autio K, et al. (2003) Structural differences between rye and wheat breads but not total fiber content may explain the lower postprandial insulin response to rye bread. Am J Clin Nutr 78, 957-964.

40. Schmitz G \& Ruebsaamen K (2010) Metabolism and atherogenic disease association of lysophosphatidylcholine. Atherosclerosis 208, $10-18$.

41. Zhao X, Peter A, Fritsche J, et al. (2009) Changes of the plasma metabolome during an oral glucose tolerance test: is there more than glucose to look at? Am J Physiol Endocrinol Metab 296, E384-E393.
42. Galili O, Versari D, Sattler KJ, et al. (2007) Early experimental obesity is associated with coronary endothelial dysfunction and oxidative stress. Am J Physiol Heart Circ Physiol 292, H904-H911.

43. Pietiläinen KH, Sysi-Aho M, Rissanen A, et al. (2007) Acquired obesity is associated with changes in the serum lipidomic profile independent of genetic effects - a monozygotic twin study. PloS ONE 2, e218.

44. Aiyar N, Disa J, Ao ZH, et al. (2007) Lysophosphatidylcholine induces inflammatory activation of human coronary artery smooth muscle cells. Mol Cell Biochem 295, 113-120. 